\title{
Relaciones trabajo-familia
}

\section{y salud en mujeres trabajadoras}

\author{
Lya Feldman, PhD, (I) Eleonora Vivas, PhD,, (I) Zoraide Lugli, MSc,, Joanmir Zaragoza, MSc, ${ }^{(1)}$ Viviola Gómez O, PhD.(2)
}

\section{Feldman L,Vivas E, Lugli Z, Zaragoza J, Gómez V. Relaciones trabajo-familia y salud en mujeres trabajadoras. Salud Publica Mex 2008;50:482-489.}

\section{Resumen}

Objetivo. Estudiar la asociación existente entre las gratiflcaciones y las interferencias generadas en la relación trabajo-familia y su impacto en la salud de mujeres trabajadoras. Material y métodos. Se utilizó un diseño no experimental correlacional. Se aplicó en Caracas,Venezuela, durante el año 2006, a un grupo de 402 mujeres trabajadoras con edades comprendidas entre 27 y 71 años, una batería de pruebas para medir relación trabajo-familia, autoestima, ansiedad, depresión, bienestar, percepción de salud y número de síntomas. Resultados. Se encontró que las gratificaciones en la relación trabajo-familia están relacionadas con un mayor bienestar y autoestima, así como con menor depresión, ansiedad y síntomas reportados. Una mayor interferencia se asoció a una mayor depresión, ansiedad y número de síntomas. Conclusiones. Los resultados permiten identificar algunos factores protectores $y$ de riesgo para la salud de las mujeres con roles múltiples y sirven de base para el diseño de planes de intervención tanto en el plano individual como organizacional.

Palabras clave: trabajo; familia; salud de la mujer;Venezuela
Feldman L,Vivas E, Lugli Z, Zaragoza J, Gómez V. Work-family relationships and health in working women. Salud Publica Mex 2008;50:482-489.

\begin{abstract}
Objective. To study the relationship between gratifications and interferences generated in the work-family relationship and its impact on working women's health. Material and Methods. A non-experimental, correlational design was used. A group of 402 working women between 27 and 71 years of age were tested in Caracas, Venezuela during 2006, to measure work-family relationship, self-esteem, anxiety, depression, wellbeing, health perception and number of symptoms. Results. It was found that the gratifications in the work-family relationship are related to better wellbeing and self-esteem, less depression, anxiety and symptom report. Presence of more interferences was associated with more depression, anxiety and number of symptoms. Conclusions. These results give an indication of protective and risk factors for multiple-role women's health and could provide some guidelines for intervention programs.
\end{abstract}

Key words: work; family; women's health;Venezuela

(I) Universidad Simón Bolívar. Departamento de Ciencia y Tecnología del Comportamiento. Caracas, Venezuela.

(2) Universidad de Los Andes. Departamento de Psicología. Bogotá,Colombia.

Fecha de recibido: 2 I de noviembre de 2007 • Fecha de aceptado: 22 de mayo de 2008 Solicitud de sobretiros: Prof. Lya Feldman.Departamento de Ciencia y Tecnología del Comportamiento. Universidad Simón Bolívar. PO Box 89.000. 1080 Caracas, Venezuela. Correo electrónico: Ifeldman@usb.ve, Ifeldman@intercable.net.ve 
$E^{n}$ las últimas décadas la participación de la mujer en el mercado laboral se ha incrementado sustancialmente. La fuerza de trabajo femenina en el mundo ha subido de 1.1 miles de millones en 1996 a 1.2 miles de millones en 2006, alcanzando una tasa de participación de 67 mujeres por cada 100 hombres. $^{1}$

Independientemente del nivel de formación académica, cada vez más mujeres con responsabilidades familiares y con hijos pequeños buscan trabajo fuera del hogar, algunas como estrategia para un mayor crecimiento y aprendizaje y otras para lograr subsistir. ${ }^{2}$ Esta realidad social ha generado la necesidad de compatibilizar el empleo con el cuidado de la familia, ${ }^{3}$ obligándola a conciliar las demandas asociadas a cada uno de sus roles y a evitar conflictos que puedan influir en su calidad de vida ${ }^{4}$ y en su salud física y mental. ${ }^{5}$

El estudio de la relación trabajo-familia ha recibido gran atención en los últimos 30 años. ${ }^{6}$ Sin embargo, el interés se ha centrado básicamente en el conflicto de roles, ${ }^{6-9}$ por lo que la investigación en esta área tiene una visión muy reducida y limitada. ${ }^{6}$

Considerar que los roles laborales y familiares pueden beneficiarse mutuamente, permite establecer nuevas direcciones de estudio de la relación trabajofamilia, tal como lo proponen Werbel y Walter, ${ }^{6}$ y romper con la tradición que concibe al trabajo como un agente que interfiere en el sistema familiar, restándole atención, recursos y energía ${ }^{6}$ a este último.

El trabajo y la familia no son esferas independientes de la vida; existe entre ellas una relación muy compleja, ${ }^{8}$ que puede ser positiva o negativa. ${ }^{10}$ La relación positiva se presenta cuando las actividades y experiencias del rol laboral se compaginan con las de los roles familiares, logrando un balance satisfactorio entre ellas. ${ }^{10}$ En este caso, la interacción es beneficiosa para ambos campos. ${ }^{6}$ Por otra parte, la relación es negativa cuando la participación en uno de estos roles obstaculiza la ejecución del otro, ${ }^{11}$ generando tensión ${ }^{10}$ y disminuyendo la satisfacción y el bienestar percibido. ${ }^{12}$

El conflicto generado en la relación negativa puede presentarse en ambas direcciones. Por ejemplo, las exigencias laborales pueden minimizar el tiempo dedicado a los familiares, ${ }^{13}$ y las dificultades en casa pueden asociarse con disminución de la productividad laboral. ${ }^{14}$

En resumen, el abordaje de las gratificaciones junto a las interferencias que se presenten al desempeñar los roles familiar y laboral representa una visión más comprehensiva del constructo de la relación trabajo-familia y permite un estudio más exhaustivo de su asociación con la calidad de vida y con la salud.

Existen contradicciones en el abordaje de la relación entre el conflicto trabajo-familia y la salud. Algunos autores plantean la hipótesis de la escasez de recursos, la cual sostiene que los individuos tienen una cantidad limitada de energía y tiempo que no les permite cumplir con todas las obligaciones y exigencias de cada rol, ${ }^{8}$ en contraposición a la hipótesis de suficiencia, la cual propone que la actividad en distintos roles puede aumentar su reservorio de energía. ${ }^{15}$ Quienes postulan la hipótesis de la escasez de recursos tienden a confirmar que el desempeño de roles múltiples produce efectos negativos sobre la salud física y mental, ${ }^{16}$ mientras que los que defienden la hipótesis de la suficiencia de recursos han hallado que el desempeño de roles incrementa el potencial físico, estimula y mejora la autoestima y brinda fuentes de apoyo y de estatus. ${ }^{16}$

Por otra parte, la mayoría de las investigaciones apoyan el planteamiento de que los conflictos trabajofamilia están asociados a diferentes niveles de estrés ${ }^{17,18}$ y estatus de salud física y mental.,19 Frone, Russell y Cooper $^{20}$ han encontrado que la interferencia trabajofamilia está asociada con altos niveles de depresión, hipertensión y pobre salud física en general. Igualmente, las interferencias trabajo-familia están vinculadas con desórdenes de ansiedad, humor y abusos de sustancias. ${ }^{21}$ Estos autores corroboran el lado negativo cuando existen interferencias en la relación.

Visto desde otra perspectiva, Greenhaus y Parasuraman $^{22}$ han hallado que el apoyo percibido en el rol laboral por parte de la organización y el recibido por los miembros de la familia, aumentan significativamente la sensación de bienestar y la satisfacción con el trabajo y el hogar.

Finalmente, considerando por un lado que los conflictos generados entre los roles labores y familiares afectan no sólo el bienestar individual, en cuanto a la salud física y mental, sino también el familiar y organizacional, ${ }^{23,24} \mathrm{y}$, por el otro, la necesidad de enfatizar nuevas direcciones de estudio de la relación trabajofamilia, no limitadas a los conflictos generados en este relación, ${ }^{6}$ el presente trabajo tiene como objetivo estudiar las gratificaciones y las interferencias generadas en la relación trabajo-familia y su impacto en la salud de mujeres trabajadoras.

\section{Material y métodos}

\section{Muestra}

Se trató de una muestra no ponderada que provino de 14 organizaciones de servicios $(n=5)$, manufactura $(n=4)$, educación $(n=2)$ y salud $(n=3)$. Cuatrocientas dos mujeres trabajadoras de Caracas, Venezuela, con edades comprendidas entre los 27 y los 71 años $(\bar{X}=41$ años, $D E=7.52)$, con diferentes niveles de instrucción $(30 \%$ 
educación secundaria, 25\% universitaria, 22\% técnica, $17 \%$ primaria y $7 \%$ ninguno) y actividad laboral (29\% secretarias, $27 \%$ enfermeras, $23 \%$ gerentes y $21 \%$ obreras), participaron de forma voluntaria en este estudio durante el año de 2006.

Doscientos sesenta y nueve de los casos muestreados $(67 \%)$ notificaron tener pareja y trescientos setenta y cinco $(93,2 \%)$ señalaron tener uno o más hijos. En cuanto al número de roles desempeñados, doscientos veinticinco mujeres $(56 \%)$ indicaron ejercer dos roles (40\% el laboral y maternal y 16\% el laboral y de pareja) y ciento setenta y siete (40\%), tres roles (laboral, pareja y maternal).

\section{Instrumentos}

Cuestionario Relación Trabajo-Familia. En la presente investigación se utilizó la versión modificada de Feldman ${ }^{25}$ del Cuestionario Relación Trabajo-Familia, originalmente diseñado por Small y Riley. ${ }^{26}$ Esta versión está constituida por 10 ítems de 4 puntos ( 4 ítems miden las posibles satisfacciones y 8 las posibles interferencias). La consistencia interna, utilizando el coeficiente alfa de Cronbach, fue de $\alpha=0,63$ para las gratificaciones y $\alpha=$ 0,82 para las interferencias.

El análisis factorial arrojó una estructura consistente con las dimensiones antes mencionadas (interferencia y satisfacción) y explica 44,5\% de la varianza total de la variable "relación trabajo-familia".

En el estudio de validez predictiva se encontró que las interferencias entre trabajo y familia se asociaban positivamente con mayor riesgo de depresión $(\mathrm{r}=0,140$ $p<0,004)$ y negativamente con bienestar $(\mathrm{r}=-0,284 p<.001)$ y autoestima $(\mathrm{r}=-0,162 p<0,001)$, mientras que la satisfacción se asoció con menores niveles de ansiedad $(\mathrm{r}=0,168$ $p<0,001) .{ }^{25}$

Número de síntomas. Se utilizó una lista de 18 síntomas físicos y psicológicos a ser respondidos en una escala dicotómica ( $1=$ No, ausencia, y $2=$ Sí, presencia), elaborada por Feldman ${ }^{25}$ a partir de una traducción y validación al castellano ${ }^{27}$ del cuestionario de Gunther ${ }^{28}$ que mide los tres componentes de la respuesta de estrés: la subjetivacognitiva, la fisiológica y la conductual-motora, obtuvo un alpha de Cronbach de 0,74.

Ansiedad. Se utilizó la sub-escala de "estado" del Inventario de Ansiedad Estado-Rasgo (STAI). ${ }^{29}$ Consta de 20 ítems de 4 opciones de respuesta, 1 (no, nunca), 4 (mucho). Estudios de confiabilidad y validez realizados con diferentes muestras y países de habla hispana han resultado satisfactorios. ${ }^{26,30,31}$

Depresión. Evaluada con un cuestionario de síntomas depresivos tomado de Pearlin, Liberman, Menaghan y Mullan, ${ }^{32}$ basado en los instrumentos de Derogatis,
Lipman, Covi y Rickles ${ }^{33}$ y Lipman, Rickless, Covi y Rikles. ${ }^{34}$ Consta de 10 ítems, respondidos en una escala de 4 puntos que va de 0 (no, nunca) a 3 (mucho). Incluye preguntas sobre humor, problemas de sueño, apetito, energía, llanto, interés en actividades cotidianas y planes para el futuro. La versión en inglés correlaciona significativamente con el Inventario de Depresión de Beck $(\mathrm{r}=0,71)$ y cuenta, además, con una consistencia interna adecuada (Alpha de Cronbach entre 0,71 y 0,85), ${ }^{35}$ igual que la adaptación en castellano en una muestra venezolana (Alpha de Cronbach $=0,72){ }^{25}$

Autoestima. Se utilizó la escala de Rosenberg ${ }^{36}$ que aborda la autoestima como autoaceptación. Consta de 10 ítems de 1 (extremadamente de acuerdo) a 4 (extremadamente en desacuerdo). En la adaptación al castellano en una muestra venezolana, se eliminaron tres ítems por correlacionar muy bajo con el puntaje total y se utiliza la escala 1 (no, nunca) a 4 (mucho). La versión en castellano cuenta con una consistencia interna apropiada (Alpha de Cronbach $=0,85){ }^{25}$

Bienestar. Medido a través de un ítem (Si usted tuviera que evaluar su nivel de bienestar en los últimos 12 meses ¿cómo lo calificaría en base a la siguiente escala?), que se responde en una escala de 10 puntos de 1 (nada satisfactorio) a 10 (muy satisfactorio).

Percepción de salud. Medida a través de un ítem (¿Cómo percibe su estado de salud en general?), que se responde en una escala de 4 puntos de 0 (muy deteriorado) a 3 (muy bueno).

\section{Procedimiento}

El proyecto se sometió al Comité de Etica del Decanato de Investigación y Desarrollo de la Universidad Simón Bolívar de Caracas, que aprobó el protocolo de investigación.

Una vez establecida la comunicación con las instituciones y organizaciones para ubicar a las participantes y obtenida la autorización de los directivos para la realización del estudio, se solicitó un listado del personal femenino que laboraba como gerente, secretaria, obrera o enfermera. Se realizó la selección al azar de las participantes, tomando en cuenta que desempeñaran el rol laboral y, al menos, uno de los siguientes roles: de pareja o de madre. Se procedió a establecer contacto con el personal seleccionado a fin de solicitar su colaboración y consentimiento informado para completar el cuestionario. Se entregó el cuestionario a las participantes, informándoles que se recogería cuatro días después. A las trabajadoras del sector obrero les fue administrado el cuestionario en forma de entrevista, fuera del horario laboral. A cada participante se le asignó un código a fin de garantizar la confidencialidad de la información. 


\section{Resultados}

Las mujeres participantes notificaron una gratificación moderada y una interferencia relativamente baja en la relación que se establece entre su trabajo y la familia. Además, en promedio presentan autoestima alta, nivel de bienestar moderado, ansiedad y depresión baja, y percepción de salud y número de síntomas notificados relativamente bajos (cuadro I).

En el cuadro II puede observarse que las gratificaciones en la relación trabajo-familia son mayores cuando la mujer desempeña un mayor número de roles y experimenta mayor autoestima y bienestar, mientras que son menores cuando es mayor el número de síntomas notificados, la ansiedad y la depresión.

Las interferencias en la relación trabajo-familia son mayores cuando es más alto el nivel educativo y el número de roles desempeñados. Igualmente, a mayor número de interferencias mayor es la ansiedad, la depresión y el número de síntomas notificados. Finalmente, esta interferencia es menor cuando hay mayores gratificaciones en esta relación y mayor número de hijos.

Para conocer las variables relacionadas con la salud, predichas por las gratificaciones, y las interferencias experimentadas por las mujeres, se utilizó análisis de regresión múltiple, previa comprobación del cumplimiento con los supuestos básicos. En el primer paso se introdujeron las variables sociodemográficas y, en el segundo, las gratificaciones y las interferencias que pueden generarse en la relación trabajo-familia. Los resultados señalan que cuando se controlan las variables sociodemográficas (número de hijos, estado civil, nivel educativo, edad y número de roles desempeñados), la relación que se establece entre el trabajo y la familia explica de forma significativa la autoestima y el bienestar (cuadro III), la depresión y la ansiedad (cuadro IV), y el número de síntomas notificados (cuadro $\mathrm{V}$ ).

Las gratificaciones e interferencias en la relación trabajo-familia explican $7.5 \%$ de la varianza total de autoestima y $6.3 \%$ del bienestar percibido por las mujeres

\section{Cuadro I}

Medias y desviaciones estándar de las variables en estudio. Caracas-Venezuela, enero 2006

\begin{tabular}{|c|c|c|c|c|}
\hline & Rango posible & Rango observado & M & $D E$ \\
\hline Relación trabajo-familia: gratificación & $0-12$ & $0-12$ & 5,67 & 2,42 \\
\hline I. Los logros en mi trabajo me han permitido enriquecer mi relación de pareja & $1-4$ & $1-4$ & 1.0525 & .99274 \\
\hline 2. Mi trabajo me ha permitido hacer nuevas amistades & $1-4$ & $1-4$ & 1.7124 & .84220 \\
\hline 3. Cumplir con mi trabajo es tan fácil como realizar tareas en el hogar & $1-4$ & $1-4$ & 1.5078 & .96948 \\
\hline 4. En mi trabajo he aprendido cosas que me sirven para ser mejor madre & $1-4$ & $1-4$ & 1.4854 & 1.00023 \\
\hline Relación trabajo-familia: interferencia & $0-24$ & $0-24$ & 7,82 & 5,69 \\
\hline I. Las horas de trabajo interfieren con el tiempo que debo dedicar a mis hijos & $1-4$ & $1-4$ & 1.0501 & .96780 \\
\hline 2. Cuando regreso a casa después del trabajo no tengo energía para dedicarme a mis labores de madre & $1-4$ & $1-4$ & .8674 & .90708 \\
\hline 3. Estoy demasiado cansada después del trabajo para ver a mis amigos con la frecuencia que quisiera & $\mathrm{I}-4$ & $1-4$ & 1.3290 & .97929 \\
\hline 4. Cuando regreso del trabajo a la casa, me faltan energías para hacer las tareas del hogar & $1-4$ & $1-4$ & 1.1218 & .97138 \\
\hline 5. Después del trabajo estoy demasiado cansada como para realizar otras actividades con mi pareja & $1-4$ & $1-4$ & .8932 & .91312 \\
\hline 6. El tiempo que dedico al trabajo interfiere con mi tiempo libre & $1-4$ & $1-4$ & .7732 & .92906 \\
\hline 7. El tiempo que dedico a la familia interfiere con mi tiempo libre & $1-4$ & $1-4$ & .6204 & .87539 \\
\hline 8. Los problemas familiares interfieren en mi trabajo & $1-4$ & $1-4$ & .3136 & .57378 \\
\hline 9. Los problemas laborales interfieren en mi relación de familia & $1-4$ & $1-4$ & .3496 & .64706 \\
\hline 10. Las preocupaciones del trabajo interfieren en mi relación de pareja & $1-4$ & $1-4$ & .4380 & .67021 \\
\hline Autoestima & $7-28$ & $7-28$ & 23,82 & 3,90 \\
\hline Ansiedad & $0-80$ & $0-50$ & 17,90 & 9,44 \\
\hline Depresión & $0-27$ & $0-18$ & 6,06 & 3,80 \\
\hline Bienestar & $0-10$ & $1-10$ & 6,68 & 1,91 \\
\hline Percepción de salud & $0-3$ & $0-3$ & 1,96 & 0,60 \\
\hline Número de síntomas & $0-18$ & $0-18$ & 5,36 & 3,62 \\
\hline
\end{tabular}


Cuadro II

Correlaciones entre la Relación tRABajo-FamiLia (GRATIFICACIONES E INTERFERENCIAS) Y LAS VARIABLES SOCIODEMOGRÁFICAS Y DE SALUD ESTUDIADAS. Caracas-Venezuela, enero 2006*

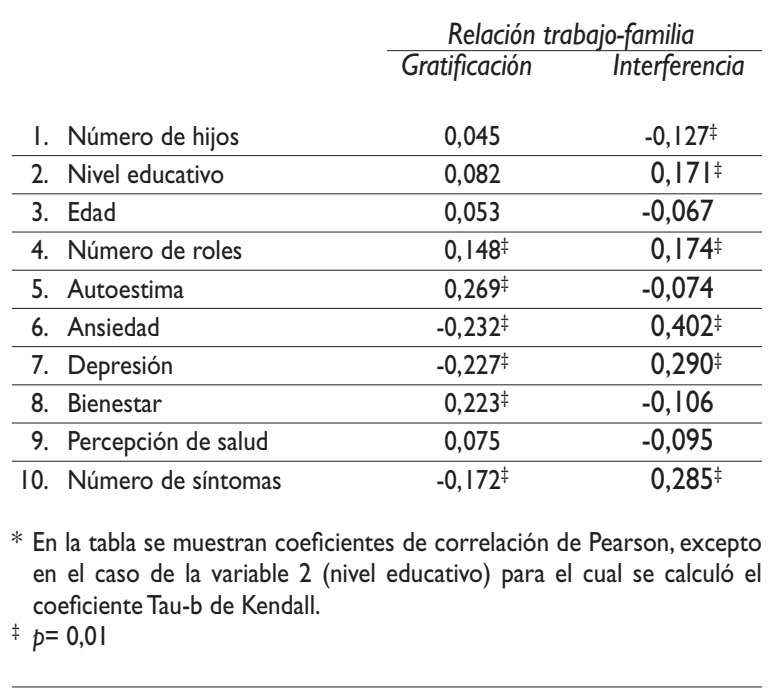

que conformaron la muestra (cuadro III). Además, se evidencia que en la medida en que existen más gratificaciones $(\beta=0,269, p=0,000 / \beta=0,244, p=0,000)$ en la relación trabajo-familia, mayor es la autoestima y el bienestar que informan las mujeres participantes de este estudio.

En el cuadro IV se muestra que las gratificaciones e interferencias en la relación trabajo-familia explican $14 \%$ de la varianza de los niveles de depresión medidos y $18.7 \%$ de los valores de ansiedad. En este caso, cuando hay menores gratificaciones $(\beta=-0,168, p=0,004 / \beta=-$ $0,156, p=0,015)$ y mayor interferencia $(\beta=0,338, p=0,000$ / $\beta=0,405, p=0,000)$, existe más depresión y la ansiedad.

Se encontró que las gratificaciones e interferencias generadas por la relación trabajo familia explican $11.7 \%$ de la varianza total, y que a menor gratificación $(\beta=-0,168$, $p=0,007)$ y mayor interferencia $(\beta=0,292 ; p=0,000)$, mayor es el número de síntomas notificados por las mujeres.

\section{Discusión}

El objetivo de esta investigación fue examinar las gratificaciones y las interferencias que pueden generarse en

Cuadro III

ANÁLISIS DE REGRESIÓN JERÁRQUICA DE LAS VARIABLES SOCIODEMOGRÁFICAS Y LAS RELACIONES TRABAJO-FAMILIA con autoestima y bienestar. Caracas-Venezuela, enero 2006

\begin{tabular}{|c|c|c|c|c|c|c|c|c|c|}
\hline Modelo & $\beta$ & $T$ & Sig. & $R$ cuadrado & $R$ cuadrado corregida & $R$ de cambio & F de cambio & Sig. & $g l$ \\
\hline \multicolumn{10}{|l|}{ Autoestima } \\
\hline I & & & & 0,014 & $-0,005$ & 0,014 & 0,745 & 0,591 & 5 \\
\hline Número de hijos & 0,041 & 0,593 & 0,553 & & & & & & \\
\hline Estado civil & $-0,063$ & $-0,930$ & 0,353 & & & & & & \\
\hline Nivel educativo & 0,114 & 1,754 & 0,081 & & & & & & \\
\hline Edad & $-0,017$ & $-0,261$ & 0,794 & & & & & & \\
\hline Número de roles & 0,036 & 0,527 & 0,599 & & & & & & \\
\hline 2 & & & & 0,088 & 0,065 & 0,075 & 11,086 & 0,001 & 2 \\
\hline Relaciones trabajo-familia: gratificación & 0,269 & 4,496 & 0,000 & & & & & & \\
\hline Relaciones trabajo-familia: interferencia & $-0,056$ & $-0,907$ & 0,365 & & & & & & \\
\hline \multicolumn{10}{|l|}{ Bienestar } \\
\hline I & & & & 0,028 & 0,010 & 0,028 & 1,562 & 0,562 & 5 \\
\hline Número de hijos & 0,008 & 0,121 & 0,904 & & & & & & \\
\hline Estado civil & $0,13 \mid$ & 1,956 & 0,052 & & & & & & \\
\hline Nivel educativo & $-0,057$ & $-0,900$ & 0,369 & & & & & & \\
\hline Edad & $-0,090$ & $-1,427$ & 0,155 & & & & & & \\
\hline Número de roles & $-0,132$ & $-1,944$ & 0,053 & & & & & & \\
\hline 2 & & & & 0,091 & 0,067 & 0,063 & 9,452 & 0,000 & 2 \\
\hline Relaciones trabajo-familia: gratificación & 0,244 & 4,087 & 0,000 & & & & & & \\
\hline Relaciones trabajo-familia: interferencia & $-0,062$ & $-1,006$ & 0,315 & & & & & & \\
\hline
\end{tabular}


Cuadro IV

ANÁLISIS DE REGRESIÓN JERÁRQUICA DE LAS VARIABLES SOCIODEMOGRÁFICAS Y LAS RELACIONES TRABAJO-FAMILIA CON DEPREsión y ANSIEDAD. Caracas-Venezuela, enero 2006

\begin{tabular}{|c|c|c|c|c|c|c|c|c|c|}
\hline Modelo & $\beta$ & $t$ & - Sig. & R cuadrado & $R$ cuadrado corregida & $R$ de cambio & F de cambio & Sig. & $g l$ \\
\hline \multicolumn{10}{|l|}{ Depresión } \\
\hline I & & & & 0,040 & 0,022 & 0,040 & 2,271 & 0,048 & 5 \\
\hline Número de hijos & 0,065 & 0,950 & 0,343 & & & & & & \\
\hline Estado civil & $-0,035$ & $-0,510$ & 0,610 & & & & & & \\
\hline Nivel educativo & $-0,122$ & $-1,906$ & 0,058 & & & & & & \\
\hline Edad & 0,091 & $\mathrm{I}, 440$ & 0,151 & & & & & & \\
\hline Número de roles & 0,041 & 0,590 & 0,556 & & & & & & \\
\hline 2 & & & & 0,181 & 0,160 & 0,141 & 23,159 & 0,000 & 2 \\
\hline Relaciones trabajo-familia: gratificación & $-0,168$ & $-2,927$ & 0,004 & & & & & & \\
\hline Relaciones trabajo-familia: interferencia & 0,338 & 5,747 & 0,000 & & & & & & \\
\hline \multicolumn{10}{|l|}{ Ansiedad } \\
\hline I & & & & 0,009 & $-0,014$ & 0,009 & 0,391 & 0,854 & 5 \\
\hline Número de hijos & $-0,075$ & $-0,960$ & 0,338 & & & & & & \\
\hline Estado civil & $-0,014$ & $-0,188$ & 0,851 & & & & & & \\
\hline Nivel educativo & 0,023 & 0,317 & 0,752 & & & & & & \\
\hline Edad & 0,069 & 0,971 & 0,333 & & & & & & \\
\hline Número de roles & 0,032 & 0,422 & 0,674 & & & & & & \\
\hline 2 & & & & 0,196 & 0,170 & 0,187 & 25,263 & 0,000 & 2 \\
\hline Relaciones trabajo-familia: gratificación & $-0,156$ & $-2,463$ & 0,015 & & & & & & \\
\hline Relaciones trabajo-familia: interferencia & 0,405 & 6,189 & 0,000 & & & & & & \\
\hline
\end{tabular}

\section{CuadroV}

ANÁLISIS DE REGRESIÓN JERÁRQUICA DE LAS VARIABLES SOCIODEMOGRÁFICAS Y LAS RELACIONES TRABAJO-FAMILIA CON EL número de síntomas reportados. Caracas-Venezuela, enero 2006

Modelo $\quad \beta \quad t \quad$ Sig. R cuadrado R cuadrado corregida R de cambio F de cambio Sig. gl

Número de síntomas

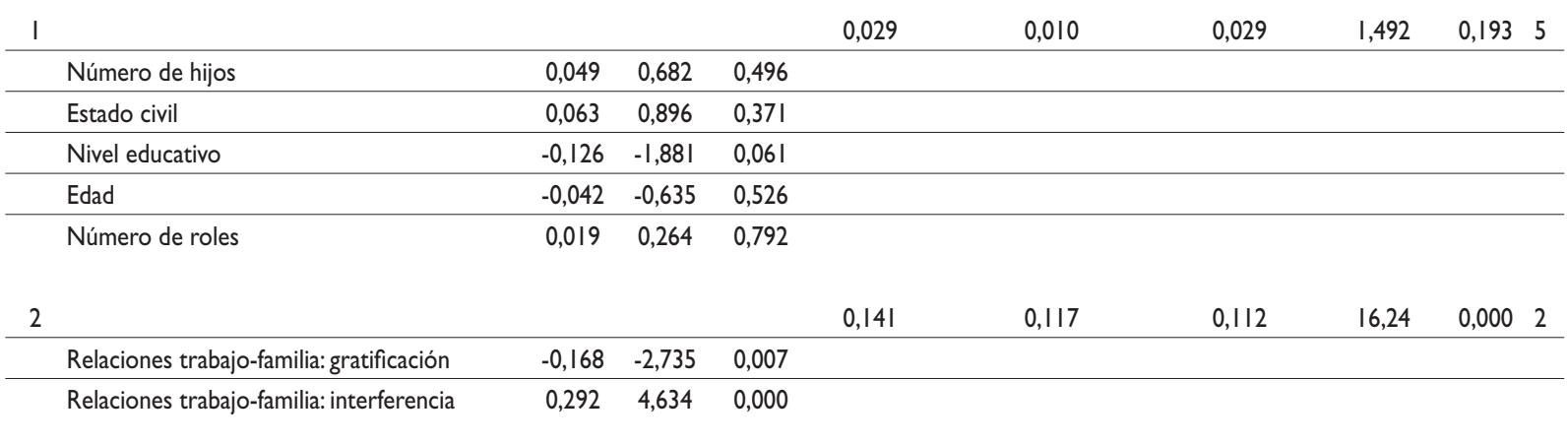


la relación trabajo-familia y su impacto sobre la salud de mujeres trabajadoras. Los resultados obtenidos indican que, en general, las participantes experimentaron más satisfacciones que interferencias en esta relación.

Las participantes notificaron un número reducido de síntomas, una buena salud autopercibida, bajos niveles de ansiedad y depresión, alta autoestima y niveles moderados de bienestar. Estos resultados, sugestivos de una buena salud física y mental, son los esperados, debido a que la muestra utilizada fue seleccionada a partir de la población general.

Con la finalidad de analizar los hallazgos sobre el impacto que tiene la relación trabajo-familia sobre la salud de las participantes, a continuación se discutirán los resultados inherentes a las gratificaciones y a las interferencias por separado.

\section{Gratificaciones}

Al examinar específicamente las gratificaciones ("enriquecer la relación de pareja con los logros laborales", "aprender cosas en el trabajo que sirven para ser mejor padre o madre", entre otros), se evidenció que éstas se relacionan, por una parte, con un mayor bienestar y autoestima y, por la otra, con menor depresión, ansiedad y síntomas reportados. En este sentido las gratificaciones pueden estar presentes por la existencia de un ambiente social enriquecido, donde es más probable que las personas experimenten una mayor autoestima y menores niveles de depresión y ansiedad. ${ }^{37}$ Estas gratificaciones también pueden relacionarse con el apoyo recibido en el hogar y en el lugar de trabajo, que repercute directamente en el bienestar percibido ${ }^{22,38}$ y la satisfacción con el trabajo y la familia. ${ }^{22}$

Este hallazgo es congruente con lo planteado por Greenhaus y Parasuraman, ${ }^{22}$ quienes señalan que el apoyo social percibido de parte de familiares y pares de la organización donde se labora genera efectos positivos que, sin duda, pueden identificarse como gratificaciones. Por otra parte, la relación encontrada entre las gratificaciones y la salud de las participantes de este estudio refuerza la postura de Werbel y Walter ${ }^{6}$ sobre el beneficio que se obtiene al lograr una mutua ganancia cuando se compaginan armoniosamente los roles que toca desempeñar tanto en el trabajo como en el hogar.

Según Kinnunen, Vermulst, Gerris y Makikangas ${ }^{24}$ la satisfacción puede reducir el nivel de estrés de las mujeres y, con ello, mejorar su bienestar. Al disminuir el nivel de estrés mejora la autoestima y se reducen los niveles de depresión; ${ }^{39}$ asimismo, es muy probable que disminuya el número de síntomas notificados, dado los efectos directos (consecuencias psicológicas y fisiológicas) e indirectos (elicitación de conductas no saludables) que tiene en la salud. ${ }^{40}$ Esta evidencia lleva a plantear que las gratificaciones pueden tener un efecto amortiguador de las experiencias negativas que podrían surgir en el desempeño de cualquiera de estos roles, ${ }^{41}$ permitiendo a las mujeres de la muestra mantener un nivel de salud adecuado.

\section{Interferencias}

Las interferencias que se encontraron como parte de la relación trabajo-familia (por ejemplo, "dificultad para realizar tareas del hogar", "dificultad para dedicar tiempo a los hijos por el cansancio producto del trabajo", entre otros), se asocian a una mayor depresión, a la presencia de ansiedad y a un mayor número de síntomas tanto físicos como psicológicos. Este resultado corrobora los hallazgos de otros estudios donde se sugiere que los conflictos o interferencias en la relación trabajo-familia están asociados negativamente con la salud mental, ${ }^{42}$ como es el caso de la depresión ${ }^{24}$ y la ansiedad. ${ }^{21,43,44} \mathrm{El}$ estado depresivo influye en el entorno, en las relaciones interpersonales e incluso en las pautas de gratificación, generando experiencias de pérdida, indefensión y estrés y afectando, por ende, la salud ${ }^{40}$ de la mujer que la experimenta. También se ha encontrado que las interferencias están asociadas con altos niveles de depresión, hipertensión, pobre salud general, ${ }^{20}$ desórdenes de humor y abusos de sustancias. ${ }^{21} \mathrm{La}$ ansiedad y la depresión son más probables en ambientes socialmente pobres, caracterizados por la presencia de conflictos entre los roles laborales y familiares, dada la falta de comunicación, comprensión y tolerancia. ${ }^{38}$

A modo de conclusión, se hace evidente que la relación que se establece entre la esfera laboral y familiar es de vital importancia para la salud física y mental autopercibida de las mujeres trabajadoras, pues repercute tanto en su autoestima y bienestar percibido, como en sus niveles de depresión, ansiedad y en el número de síntomas notificados. Al tomar en cuenta el número creciente de mujeres que se integran al mercado laboral en el mundo, en especial aquellas con hijos pequeños, se requiere prestar una mayor atención a esta relación. Por otra parte, se reafirma la posición que postula la complementación de roles y la necesidad de incorporar en los estudios sobre roles múltiples tanto los aspectos positivos como los negativos, a fin de poder tener una visión mas comprehensiva de esta interacción.

La identificación de los elementos gratificantes y de las interferencias que se generan a partir de la interacción trabajo-familia serviría como base para el diseño de programas de intervención dirigidos a mejorar la relación trabajo-familia y sus repercusiones en 
los planos personal, familiar, laboral y organizacional. Adicionalmente, el análisis por puesto podría ayudar a precisar cuáles son los grupos ocupacionales que perciben mayores interferencias o gratificaciones en relación con la salud, lo cual puede ser objeto de futuras investigaciones.

\section{Referencias}

I. OIT.Tendencias Mundiales del Empleo de las Mujeres. [Internet]. Ginebra: Organización Mundial del Trabajo 2007 [consultado 2007 marzo 7]. Disponible en: http://www.ilo.org/public/english/employment/strat/ download/getb07sp.pdf

2. Monteverde GG. ¿Por qué trabajan las mujeres? Revista El Colegio de Sonora [Revista en línea] 1996; 7(12): [161-167] [consultado 2007 mayo I0] Disponible en: http://lanic.utexas.edu/project/etext/colson/I2/I2_6.pdf 3. Martínez C,Vera JJ, Paterna C,Alcázar AR.Antecedentes del conflicto interrol y su relación con el autoesquema de género. An Psicol 2002; I8(2): 305-317.

4. Grennhaus JH, Collins KM, Shaw, JD. The relation between work-family balance and quality of life. JVocat Behav 2003; 63: 510-53I.

5. Mesmer-Magnus IR,Viswesvaran C. Convergence between measures of work-to-family and family-to-work conflict: A metanalytic examination life. JVocat Behav 2005; 67: 215-232

6. Werbel J,Walter $\mathrm{MH}$. Changing views of work and family roles. A symbiotic perspective. Hum Resour Manage Rev 2002; 12: 293-298.

7. Byron K.A meta-analytic review of work-family conflict and its antecedents. JVocat Behav 2005; 67:169-198.

8. Grennhaus JH, Powell GN.When work and family collide: deciding between competing role demands. Organ Behav Hum Decis Process 2003; 90: 291-303.

9. Stoeva AZ, Chiu RK, Greenhaus JH. Negative affectivity, role stress, and work-family conflict. JVocat Behav 2002;60:I-I6.

10. Eckenrode J, Gore S. Stress between work and family. New York: Plenum Press, 1990.

I I. Burke RJ, Greenglass ER.Work-family conflict, spouse support, and nursing staff well-being during organizational restructuring.J Occup Health Psychol 1999; 4(4): 327-336.

12. Frone MR, Russell M, Cooper ML. Antecedent and outcomes of workfamily conflict:Testing a model of the work-family interface. J Appl Psychol 199|; 77: 65-78

13. Hochschield A. The time bind: when work becomes home and home becomes work. New York: Metropolitan Books, 1997.

14. Eby LT, Casper W], Lockwood A, Bordeaux C, Brinley A. Work and family research in $\mathrm{IO} / \mathrm{OB}$. Content analysis and review of the literature (1980-2002). JVocat Behav 2005; 8: I24-197.

15. Marks SR. Multiple roles and role strain: some notes on human energy, time and commitment. Am Sociol Rev 1977; 42: 921-936.

16. Baruch GK, Barnett RC. Role quality, multiple role involvement, and psychological well-being in midlife women.J Pers Soc Psychol I986; 5 I (3): 983-992.

17. Burke RJ, Greenglass ER. Work-family conflict, spouse support, and nursing staff well-being during organizational restructuring.J Occup Health Psychol 1999; 4(4): 327-336.

18. Barnett RC, Gareis KC, Brennan RT. Fit as a mediator of the relationship between work hours and burnout. J Occup Health Psychol 1999; 4(4): 307-317

19. Bedeian AG, Burke BG, Moffett RG. Outcomes of work-family conflict among married male and female professionals. J Manage 1988; 14:475-491. 20. Frone MR, Russell M, Cooper ML. Relation of work-family conflict to health outcomes: a four year longitudinal study of employed parents. J Organ Behav 1997; 70: 325-335.
2I. Frone MR. Interpersonal conflict at work and psychological outcomes: Testing a model among young workers. J Occup Health Psychol 2000; 5(2): 246-255.

22. Greenhaus $\mathrm{H}$, Parasuraman S. Work-family conflict, social support and well-being. En: Davidson MJ, Burke RJ, eds. Women in management; current research issues. London: Paul Chapman, 1994:213-229.

23. Allen TD, Herst DE, Bruck CS, Sutton M. Consequences associated with work-to-family conflict:A review and agenda for future research.] Occup Health Psychol 2000; 5: 278-308.

24. Kinnunen V,Vermulst A, Gerris J, Makikangas A. Work family conflict and its relations to well-being. The role of personality as a moderating factor. Pers Individ Dif 2003; 35: 1669-1683.

25. Feldman L. Social roles, psychological factors and health in Venezuelan working women [dissertation]. Southampton: School of Health Professions and Rehabilitation Sciences Faculty of Medicine, Health and Biological Sciences - University of Southampton. United Kingdom: University of Southampton, 2001

26. Small SA, Riley D. Toward a multidimensional of work spillover into family life.J Marriage Fam 1990; 52:5I-61.

27. Canino E, Groeger C, Robles J.Adaptación del cuestionario excitabilidad individual: confiabilidad, análisis de ítems y estructura factorial.Argos 1994; 19: 43-66

28. Gunther K. Stress y conflictos: métodos de superación. Madrid: Paraninfo, 1980.

29. Spielberger C, Gorsuch R, Luchene R, Vagg P, Jacobs C. Manual for the state-trait anxiety inventory. Palo Alto: Consulting Psychologists Press, 1983.

30. Díaz-Guerrero R, Spielberger C. IDARE: Inventario de ansiedad: Rasgo-Estado. Manual e instructivo. STAl:Anxiety inventory: State and trait. (Manual). México: El Manual Moderno, 1975.

31. Granell E, Feldman L. Un inventario de temores para estudiantes universitarios: Un estudio normativo. Psicología |98|; 8 (2 y 3): I55-|72. 32. Pearlin L, Liberman M, Menaghan E, Mullan J.The stress process.J Health Soc Behav I98I; 22: 337-356.

33. Derogatis L, Lipman R, Covi L, Rickles K. Neurotic symptom dimension. Arch Gen Psychiatry 197I; 24(5): 454-464.

34. Lipman R, Rickless K, Covi L, Derogatis L, Uhlenhuth E. Factors of symptoms distress Doctor ratings of anxious neurotic outpatients. Arch Gen Psychiatry 1969; 21(3): 328-358.

35. Horsten M,Wamala SP,Vingerhoets A, Orth-Gomer K. Depressive symptoms, social support, and lipid profile in healthy middle-aged women. Psychosom Med 1997; 59:521-528.

36. Rosenberg M. Society and the adolescent self-image. Princeton: Princeton University Press, 1965

37. Repetti RL. Individual and common components of the social environment at work and psychological well-being.J Pers Soc Psychol 1987; 52: 710-720

38. Carlson DS, Perrewé PL. The role of social support in the stressorstraing relationship: an examination of work-family conflict. J Manag 1999; 25: $513-540$

39. Schwartzberg NS, Dytell RS. Dual earner families: The importance of work stress and family stress for psychological well-being.J Occup Health Psychol 1996; I:21 I-223.

40. Belloch A, Sandín B, Ramos F. Manual de Psicopatología. España: McGraw Hill, 1995.

4I. Barnett RC, Hyde JS. Women, men, work, and family: an expansionist theory.Am Psychol 200I; 56: 78I-796.

42. Curbow B, McDonnell K, Spratt K, Griffin J,Agnew J. Development of the work-family interface scale. Early Childhood Res Q 2003; 18: 310-330. 43. Grzywacz J, Bass B.Work, family, and mental health: testing different models of work-family fit.J Marriage Fam 2003; 65: 248-262.

44. Greenhaus JH, Buttel NJ. Sources of conflict between work and family roles.Acad Manage Rev 1985; 10: 76-88. 